\title{
Jitter Decomposition in Ring Oscillators *
}

\author{
Qingqi Dou \\ Computer Engineering Research Center \\ The University of Texas at Austin \\ Austin, TX 78712 \\ e-mail: qdou@cerc.utexas.edu
}

\author{
Jacob A. Abraham \\ Computer Engineering Research Center \\ The University of Texas at Austin \\ Austin, TX 78712 \\ e-mail: jaa@cerc.utexas.edu
}

\begin{abstract}
It is important to separate random jitter from deterministic jitter to quantify their contributions to the total jitter. This paper identifies the limitations of the existing methodologies for jitter decomposition, and develops a new and efficient approach using time lag correlation functions to decompose different jitter components. The theory of the approach is developed and it is applied to a ring oscillator simulated in a 0.6-um AMI CMOS process. Results show good agreement between the theory and hspice simulation.
\end{abstract}

\section{INTRODUCTION}

Ring oscillators have been integrated into many digital and communication systems due to their low area, low power and fast response time. They are used in serial links, disk-drive read channels in data communications, Clock and Data Recovery (CDR) circuitry in optical communication networks, and frequency synthesizers in wireless communications. In such applications, jitter is one of the most important parameters affecting the Bit-Error Rate (BER). BER is determined by the signal integrity, which is quantified by Signal-to- Noise Ratio (SNR) and Total Jitter (TJ) [3, 4]. Therefore, both signal amplitude noise and jitter magnitude must comply with specifications in the communication standards for a certain BER level.

One of the greatest challenges in integrating all circuitry on the same chip is in the placement of sensitive analog circuits (e.g., ring oscillators) and large digital blocks to meet the signal integrity requirements. With the global supply and ground busses, ring oscillators may experience performance degradation due to the substrate and supply noise. As demonstrated in $[2,1]$, the noise manifests itself as jitter at the output of ring oscillators. Though the jitter magnitude induced by the supply and substrate noise far exceeds that introduced by the device electronic noise, its contribution to the TJ is weighted approximately ten times less [4]. Therefore, jitter decomposition is necessary for accurately deriving $\mathrm{TJ}$ and BER.

This paper presents a new methodology for jitter decomposition using Time Lag Correlation (TLC) functions. TLC treats jitter in its original form, as a time series, resulting in good accuracy in the decomposition.

In section ii, we define and analyze the problems of jitter

* This work was supported by the National Science Foundation under Grant No. CCR-0325371 and in part by Subcontract No. SA3271JB from UC Berkeley under prime Contract 2003-DT-660 from Microelectronic Advanced Research Corporation (MARCO). decomposition in ring oscillators. The limitations of conventional methods are discussed in section iii. In section iv, the basic theory of TLC functions is presented and equations using them for jitter decomposition are derived. The generality of the theory is verified with different assumptions in section v. In section vi, the developed approach is applied to seperate Random Jitter (RJ) from Sinusoid Jitter (SJ) injected by the thermal and supply noise respectively, in a ring oscillator and the simulation results are presented.

\section{PROBLEM DESCRIPTION}

\section{A. Jitter Definitions}

Jitter is a general term; it can include absolute jitter, cycle jitter or cycle-to-cycle jitter (Figure 1). Cycle jitter is defined as the deviation of a signals $n$th timing event, $t_{n}$, from its intended (ideal) occurrence in time. The $n$th period is then defined as $T_{n}=t_{n+1}-t_{n}$, while the ideal point is normally the minus-to-plus zero crossing of the signal, which is determined by the period, T. Then, the cycle jitter is defined as $j_{n}=T_{n}-\bar{T}$, where $\bar{T}$ is the mean period, the average value of the measured periods.

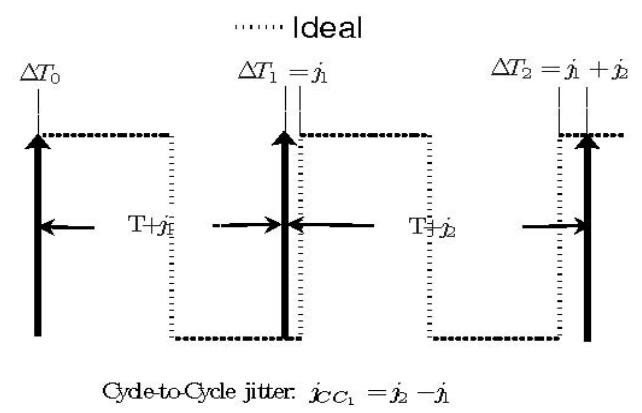

Fig. 1. Absolute jitter, cycle jitter, and cycle-to-cycle jitter

Absolute jitter defined [9] as

$$
\Delta T_{N}=\sum_{n=1}^{N} j_{n}
$$

and is often used to quantify the jitter of Phase-Locked Loops (PLLs) or to derive the BER. This is, in fact, an integral of the 
cycle jitter; thus, the variance of $\Delta T_{N}$ diverges with time [2]. Therefore, this is not a good indicator for jitter analysis.

Cycle-to-cycle jitter is given by

$$
j_{C C_{n}}=j_{n+1}-j_{n}
$$

The difference between the cycle jitter and the cycle-to-cycle jitter is that the former compares the measured period with the mean period while the latter compares with the preceding period. Hence, cycle-to-cycle jitter is the first difference of the cycle jitter, representing the short-term dynamics of the period, while cycle jitter contains no information about the dynamics. Though cycle jitter is used here, both $\Delta T_{N}$ and $j_{C C_{n}}$ can be derived similarly.

In general, the jitter distributions encountered contain both bounded and unbounded random components as well as deterministic components. RJ is assumed to be a Gaussian distribution with a Root-Mean-Square (RMS) value while Deterministic Jitter (DJ) is represented by its Peak-to-Peak (PP) value. DJ can be further categorized according to the source of the jitter. For example, power switching noise leads to SJ; mismatch in the differential circuits gives Duty Cycle Distortion (DCD); while bandwidth limited channel introduces Intersymbol Symbol Interference (ISI), etc.

Provided that RJ and DJ are independent variables, the sum function in the time domain is equal to the convolution of the RJ and the DJ distribution [7]. There are several reasons to separate the different jitter components. A simple case of TJ consisting of an RJ with an RMS value of $0.5 \mathrm{ps}$ and an SJ with a PP value of $5 \sqrt{2}$ will be used to illustrate the problem. The problem encountered is that neither the RMS nor the PP value of the PDF represents either the RMS of the RJ or the PP value of the SJ (Tab. I). The PP value of the convoluted histogram is no longer constant because the RJ is included. Also, the RMS value of the convoluted histogram is not that of the RJ component.

Another reason to separate the RJ and the DJ is that they accumulate differently in a serial link. TJ is actually the sum of $P P_{R J}$ and $P P_{D J}$, where $P P_{R J}=K_{\sigma} * R M S_{R J} . K_{\sigma}$ denotes a weight factor associated with different BER boundaries [8] (Tab. II).

TABLE I

RMS AND PP OF A CONVOLUTED JITTER (PS)

\begin{tabular}{|c|c|c|}
\hline Sample Size & RMS & PP \\
\hline 1000 & 5.02 & 8.21 \\
11000 & 5.02 & 8.45 \\
101000 & 5.03 & 8.85 \\
\hline
\end{tabular}

\section{B. Jitter Injection in a Ring Oscillator}

Fig. 2 illustrates a three-stage ring oscillator, where all the unit delay cells share the power supply. SJ is introduced by coupling a sinusoid noise to the power supply voltage (Fig. 3) while RJ is injected through the thermal noise [10]. The jitter resulting from the simultaneous injection of the RJ and $\mathrm{SJ}$ is demonstrated in Fig. 4.
TABLE II

WEIGHT FACTOR $K_{\sigma}$ VS. BER

\begin{tabular}{|c|c|c|c|}
\hline BER & $K_{\sigma}$ & BER & $K_{\sigma}$ \\
\hline $10^{-4}$ & 7.44 & $10^{-11}$ & 13.41 \\
$10^{-5}$ & 8.53 & $10^{-12}$ & 14.07 \\
$10^{-6}$ & 9.51 & $10^{-13}$ & 14.70 \\
$10^{-7}$ & 10.40 & $10^{-14}$ & 15.30 \\
$10^{-8}$ & 11.22 & $10^{-15}$ & 15.80 \\
$10^{-9}$ & 12.00 & $10^{-16}$ & 16.44 \\
$10^{-10}$ & 12.72 & $10^{-17}$ & 16.93 \\
\hline
\end{tabular}

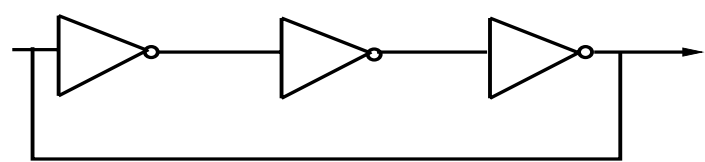

Fig. 2. Three-stage ring oscillator

\section{Limitations of CONVENTIONAL Methods}

Histogram-based analysis specifies only the TJ through Peak-to-Peak (PP) or Root-Mean-Square (RMS) value, ignoring the parameters of the deterministic jitter, such as the frequency of the SJ. Since SJ is a function of time, the PDF, a simple counting of events, contains no knowledge of how the events evolve in time. The events could have happened in ascending order, descending order, or in a completely random fashion. Figure 5 compares two TJs both consisting of a RJ with a RMS value of 5ps and an SJ with a PP value of $5 \sqrt{2}$ but different frequencies. The two histograms differ little even with a large sample size.

The TailFit algorithm [5], extracts the RMS value of the RJ from the tail of the PDF and defines the difference between the two peaks in the histogram as the PP value of the DJ (Figure 6). However, it fails when the RMS value is comparable to the PP value. Figure 6 demonstrates that the value of the DJ cannot be extracted as there is only one peak. Even more samples do not help.

Generally, the time evolution of a process can be quantified by the power spectrum, providing an estimation of the contri-

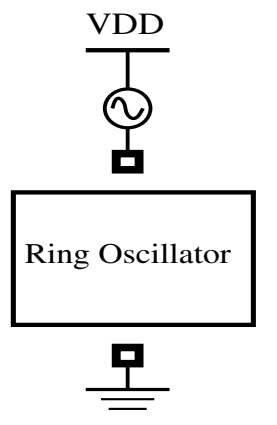

Fig. 3. SJ is injected by the supply noise 


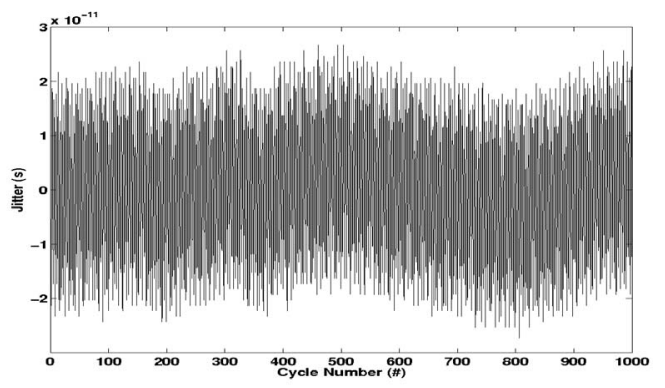

Fig. 4. Jitter induced by thermal and supply noise

bution of each frequency to the total variance. Spectral analysis normally gives good precision in estimating the frequency of the SJs [6]. However, it is not straightforward to deal with the time series and there still exists several issues, such as, how to separate jitter with SJ, DCD, and other deterministic components; how to detect high-frequency jitter; and how to overcome the aliasing problem.

Another way to view the time evolution is in terms of TLC. This is efficient for our purposes because each component of jitter evolves differently with time. For example, RJ, as a random variable, has little correlation with its lagged time series while SJ has strong correlation with its lagged version. It will be illustrated in Section IV that it is possible to separate RJ, multiple SJs and DCD by their different functions of the TLC.
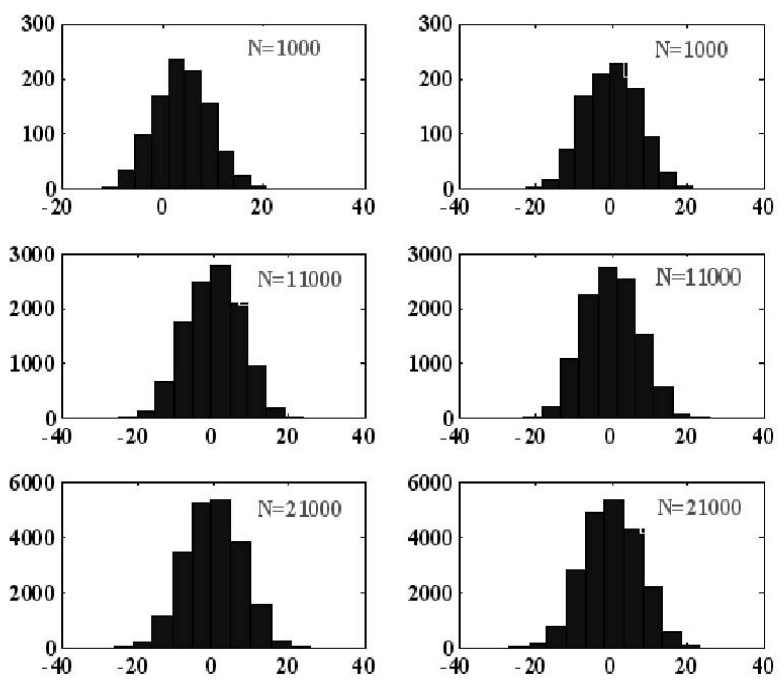

Fig. 5. Dependence of PDF on the sample size and its inability to extract the frequency of $\mathrm{SJ}, f_{m}$

\section{The Proposed Methods}

This section illustrates how the TLC functions of different jitter components differ. and how the TLC function of the time series for jitter helps to separate SJ, RJ, and DCD. The basic theory of TLC and its efficiency in separating RJ and DJ will be demonstrated in the following. Jitter as a time series can be

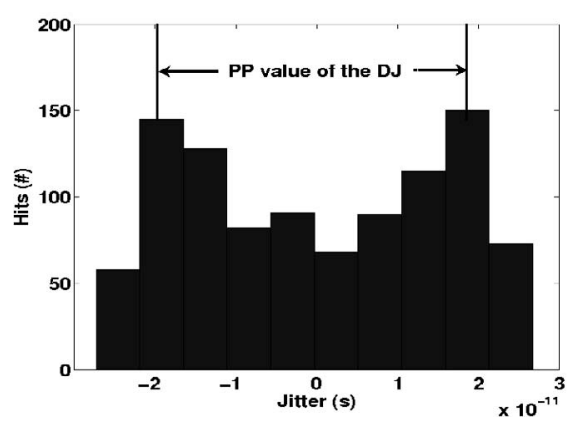

Fig. 6. Histogram-based method

represented by TLC, which is defined as

$$
C_{j}(m)=\lim _{N \rightarrow \infty} \frac{1}{N} \sum_{n=1}^{N}\left(j_{n+m} j_{n}\right),
$$

where $C_{j}(m)$ is the TLC of the cycle jitter, defined as the correlation of a variable with itself over $m$ successive time lags with a sample size of N. Ideally, a random variable only has autocorrelation when time lag is zero

$$
C_{j}(0)=\sigma_{R J}^{2}
$$

which means that the TLC with a zero argument is the variance of the cycle jitter. The TLC drops rapidly as it departs from zero, finally approaching zero when the time lags approaches infinity (Fig. 7).

The RMS of the cycle-to-cycle jitter for a RJ can also be expressed by the TLC of the cycle jitter.

$$
\sigma_{C C}^{2}=\lim _{N \rightarrow \infty} \frac{1}{N} \sum_{n=1}^{N}\left(j_{n+m}-j_{n}\right)^{2}
$$

This indicates that the variance will double if two Gaussian variables are subtracted with identical means and standard deviations as $C_{j}(1)$ is nearly zero here.

Similarly, the cycle jitter and the cycle-to-cycle jitter of an SJ can also be expressed by the TLC, with all values being the PP values. Assume the SJ to be

$$
j(m)=P P * \cos \left(\frac{m \varpi_{m}}{f_{0}}\right),
$$

where $\varpi_{m}$ is the angular frequency of the SJ and $f_{0}$ is the signal frequency. The time lag number is represented by $\mathrm{m}$, which corresponds to a time lag of $\frac{m}{f_{0}}$. Then the TLC of the SJ is

$$
\begin{gathered}
C_{j}(m)=\lim _{N \rightarrow \infty} \frac{1}{N} \sum_{n=1}^{N}\left(j_{n+m} j_{n}\right) \\
=\frac{P P^{2}}{2} \cos \left(\frac{m \varpi_{m}}{f_{0}}\right) .
\end{gathered}
$$

When $\mathrm{m}=0$,

$$
C_{j}(0)=\frac{P P^{2}}{2}=\sigma_{S J}^{2}
$$


where $\sigma_{S J}$ is the RMS of the SJ. Equation 8 represents the fact that the PP value of the SJ is $\sqrt{2} \sigma_{S J}$, while Equation 7 indicates that the TLC of SJ is dependent upon the PP value and the frequency of SJ (Fig. 8).

If the SJ and the RJ coexist in the cycle jitter, the TLCs will filter out the RJ when the number of time lag is nonzero.

$$
\begin{gathered}
C_{j}(0)=\frac{P P^{2}}{2}+\sigma_{R J}^{2} \\
C_{j}(m)=\frac{P P^{2}}{2} \cos \left(\frac{m \varpi_{m}}{f_{0}}\right)
\end{gathered}
$$

, where $\mathrm{m} \neq 0$. Equations 9 and 10 indicate that only three autocorrelation values $C_{j}(0), C_{j}\left(m_{1}\right)$, and $C_{j}\left(m_{2}\right)$ are necessary to derive the RMS value of the RJ, PP value of the SJ and the frequency of the $\mathrm{SJ}, f_{m}$.

If multiple SJs are considered, for example, two SJ, $j_{s_{1}}$ and $j_{s_{2}}$, then the TLC will be

$$
C_{j}(0)=\frac{P P_{1}^{2}}{2}+\frac{P P_{2}^{2}}{2}+\sigma_{R J}^{2}
$$

and

$$
\begin{aligned}
C_{j}(m) & =\frac{P P_{1}^{2}}{2} \cos \left(\frac{m \varpi_{m_{1}}}{f_{0}}\right) \\
& +\frac{P P_{2}^{2}}{2} \cos \left(\frac{m \varpi_{m_{2}}}{f_{0}}\right)
\end{aligned}
$$

, where $\mathrm{m} \neq 0$. This indicates that five $C_{j}(m)$ values are necessary to derive $P P_{1}, P P_{2}, \varpi_{m 1}$ and $\varpi_{m 2}$ of the SJs and the RMS value of the RJ.

It is still possible to separate the different components of jitter if it consists of RJ, SJ, and DCD as they differ in their TLCs, for example, for a single SJ, DCD and RJ,

$$
\begin{aligned}
C_{j}(0) & =\frac{P P^{2}}{2}+\sigma_{R J}^{2}+\frac{D C D^{2}}{4}, \\
C_{j}(2 k+2) & =\frac{P P^{2}}{2} \cos \left(\frac{2 k \varpi_{m}}{2 f_{0}}\right)+\frac{D C D^{2}}{4},
\end{aligned}
$$

and

$$
\begin{aligned}
C_{j}(2 k+1) & =\frac{P P^{2}}{2} \cos \left(\frac{(2 k+1) \varpi_{m}}{f_{0}}\right) \\
& -\frac{D C D^{2}}{4} .
\end{aligned}
$$

, where $\mathrm{k}=0,1,2, \cdots$.

\section{VERIFICATION OF THE THEORY}

This section presents a case of jitter composed of an SJ and an RJ to verify the proposed method. The key factors, the sample size and the error are studied with SJ frequency, $f_{m}$; system central frequency, $f_{0}$; the ratio of the RMS value of RJ to the $\mathrm{PP}$ value of $\mathrm{SJ}, \alpha$; and the ratio of the TJ to a bit period, i.e., Unit Interval (UI).

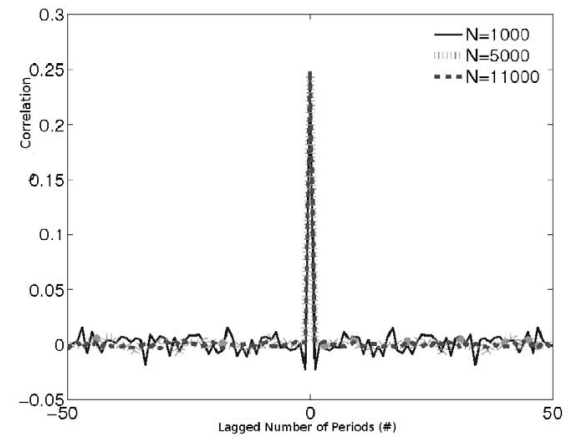

Fig. 7. TLC of RJ

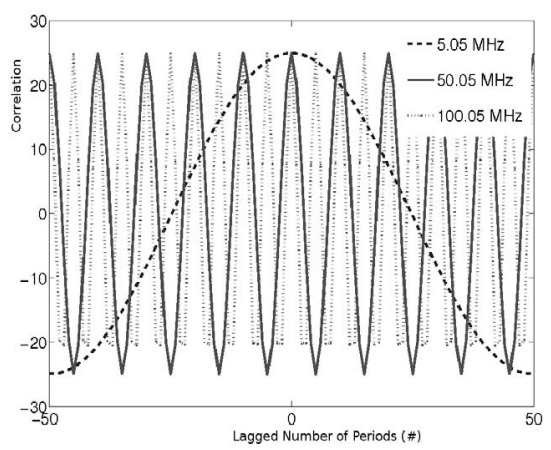

Fig. 8. TLC of SJ with same PP at different frequencies

\section{A. Total Jitter versus Unit Interval}

The increasing importance of jitter in high data rate systems derives from the increasing weight of its ratio to the bit period. This section investigates whether the ratio of the TJ to UI will affect the sample size and the error in the algorithm. Specifically, for a system with a central frequency of $1 \mathrm{GHz}$, four cases are studied: $0.05 \mathrm{UI}, 0.1 \mathrm{UI}, 0.15 \mathrm{UI}$ and $0.2 \mathrm{UI}$ while the other two factors, $f_{m}$ and $\alpha$, are assumed constant. For this, we make $f_{m}=420 \mathrm{MHz}$ and $\alpha=10$. The results in Tab. III show good accuracy, even when the sample size is only 1000.

\section{B. Root Mean Square Value of Random Jitter versus Peak-to- Peak Value of Sinusoid Jitter}

Typically, the RMS of RJ is approximately ten times less than the PP of SJ [2], thus, ignored by the designers. However, as Tab. II illustrates, the weight factor, $K_{\sigma}$, distinguishes $\mathrm{RJ}$ in the TJ. Therefore, it is necessary to discuss whether the method developed here is robust to different values of $\alpha$. Four cases will be studied: $\alpha=1,5,10$ and 20 while assuming other parameters are held constant. For this, we make $f_{0}=1 \mathrm{GHz}$, $f_{m}=420 \mathrm{MHz}, K_{\sigma}=14$, and TJ $=0.1 \mathrm{UI}$.

Results illustrate that the error of extracting the PP value of the SJ is inversely proportional to $\alpha$ (Fig. 9), while it is the opposite for the RJ parameters. 
TABLE III

$f_{m}$ Percentage Error $(\%)$ vs. TJ

\begin{tabular}{|c|c|c|c|c|}
\hline Sample Size & $0.05 \mathrm{UI}$ & $0.1 \mathrm{UI}$ & $0.15 \mathrm{UI}$ & $0.2 \mathrm{UI}$ \\
\hline 1000 & 0.024 & 0.014 & 0.013 & 0.01 \\
5000 & 0.003 & 0.006 & 0.005 & 0.005 \\
\hline
\end{tabular}

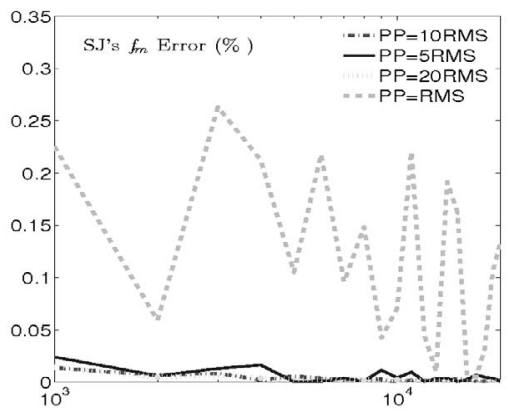

Fig. 9. $f_{m}$ Error (\%) vs. Sample Size

\section{C. $f_{m}$ of Sinusoid Jitter versus System Central Frequency $f_{0}$}

For the frequency case, as the $\mathrm{SJ}$ is sampled by $f_{0}$, it is necessary to specify the frequency of the SJ, $f_{m}$ within a frequency range, such as $0 \sim \frac{f_{0}}{2}, \frac{f_{0}}{2} \sim f_{0}, \ldots, \frac{k f_{0}}{2} \sim \frac{(k+1) f_{0}}{2}$ to overcome the aliasing problem. It is also required to investigate whether this method has problem dealing with high or low $f_{m}$ or $f_{0}$.

Tab. IV (with sample size $=3000$ ) shows that the error is independent of the $f_{m}$ frequency ranges and $f_{0}$, even when $f_{0}$ increases to $10 \mathrm{GHz}$.

\section{Simulation Results IN Ring OSCILlatoR}

A current starved three-stage ring oscillator built with the unit delay cell shown in Fig. 10 is simulated in the 0.6-um AMI CMOS process. The oscillation frequency and the voltage swing are controlled by the pMOS current source and the diode connected nMOS device, respectively. This circuit oscillates at $341 \mathrm{MHz}$ without perturbation under a supply voltage of $3 \mathrm{~V}$ and a control voltage of $1.5 \mathrm{~V}$.

TABLE IV

Percentage ERror $(\%)$ vs. $f_{0}(\mathrm{~Hz})$

\begin{tabular}{|l||l|l||l|l|l|l|}
\hline \multicolumn{1}{|c||}{} & \multicolumn{3}{l|}{$f_{m}=0.0112 f_{0}$} & \multicolumn{3}{l|}{$f_{m}=0.112 f_{0}$} \\
\cline { 2 - 7 }$f_{0}$ & $f_{m}$ & PP & RMS & $f_{m}$ & PP & RMS \\
\hline \hline $10 \mathrm{M}$ & 2.48 & -0.02 & 1.23 & 0.004 & -0.03 & 1.42 \\
$10 \mathrm{G}$ & 2.43 & -0.05 & 1.08 & 0.006 & -0.03 & 1.32 \\
\hline \hline \multirow{4}{*}{$f_{0}$} & $f_{m}=1.012 f_{0}$ & \multicolumn{4}{c|}{$f_{m}=10.012 f_{0}$} \\
\cline { 2 - 7 } & $f_{m}$ & PP & RMS & $f_{m}$ & PP & RMS \\
\hline \hline $10 \mathrm{M}$ & 0.01 & 0.02 & 1.05 & 0.002 & 0.04 & 1.34 \\
$10 \mathrm{G}$ & 0.02 & -0.04 & 0.57 & 0.002 & -0.01 & 1.89 \\
\hline
\end{tabular}

Three runs are required to verify the accuracy of the proposed method. The first run is a single injection of thermal noise while the second is a single injection of supply noise. The third run injects both the noise sources. The golden values of the SJ and RJ are extracted from the first two runs and are referred to as the actual values. The linear superposition of the thermal and supply noise sources is verified through the Discrete Fourier Transform (DFT) of the output jitter signal. The plot in Fig. 11 indicates that the modulation between the thermal noise and the supply sinusoid noise is negligible. The results also show that the white noise floor of the SJ is around $15 \mathrm{~dB}$ below that of the RJ. Thus, it is assumed that the thermal noise causes RJ while the supply sinusoid noise gives rise to SJ. Therefore, the total induced jitter can be treated simply as a single SJ superimposed with RJ.

To investigate the effects of $f_{m}$ and the $\alpha$ ratio on the accuracy of the proposed method, two levels of the frequencies of the $\mathrm{SJ}\left(f_{m}=100 \mathrm{MHz}\right.$ and $200 \mathrm{MHz}$ ) are combined with two different amplitudes of the sinusoid noise, $2.5 \mathrm{mV}$ and 1.25 $\mathrm{mV}$ as the test cases. The results in Tab. V demonstrate that the accuracy of the proposed method is not highly dependent on the $f_{m}$ while a smaller $\alpha$ gives a more accurate RMS value of the RJ. These observations are consistent with the conclusions drawn in section $\mathrm{V}$.

Fig. 12 illustrates that the PP value of the SJ in cycle jitter is almost linearly dependent on the noise amplitude, and mostly independent of $f_{m}$, as demonstrated in [2]. The results at the upper and lower parts of the plot correspond to the cases in which the supply sinusoid noise of $2.5 \mathrm{mV}$ and $1.25 \mathrm{mV}$ are injected, respectively. The results show that the proposed method extracts the PP values of the SJ with a maximum error of $8 \%$ (sample size $=1000$ ) while the histogram-based method fails as the two peaks for predicting the PP value of the SJ converge. Fig. 13 indicates that the histogram-based method is only applicable to the cases in which the PP value of the SJ far exceeds the RMS value of the RJ.

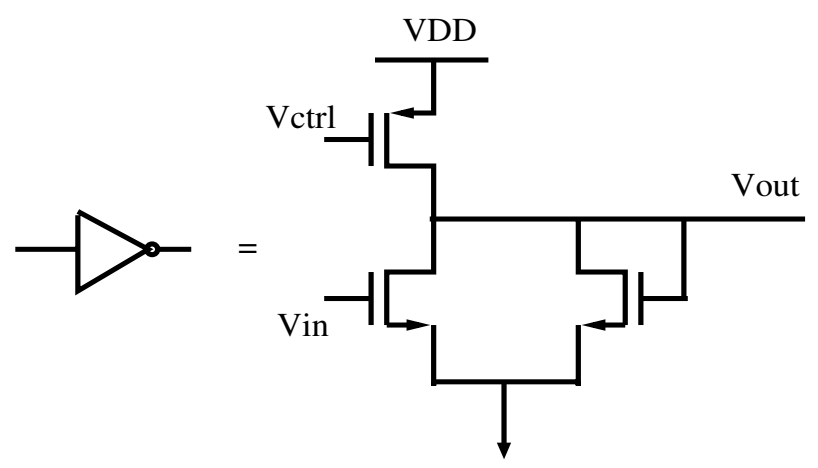

Fig. 10. Unit delay cell

\section{CONCLUSIONS AND FUTURE WORK}

We have demonstrated an efficient technique for jitter decomposition using time lag correlation functions. This method extracts the frequency of the $\mathbf{S J}\left(f_{m}\right)$ and is applicable to any $\alpha$ ratio, overcoming the limitations of the histogram-based method. This approach was applied to a ring oscillator, and 


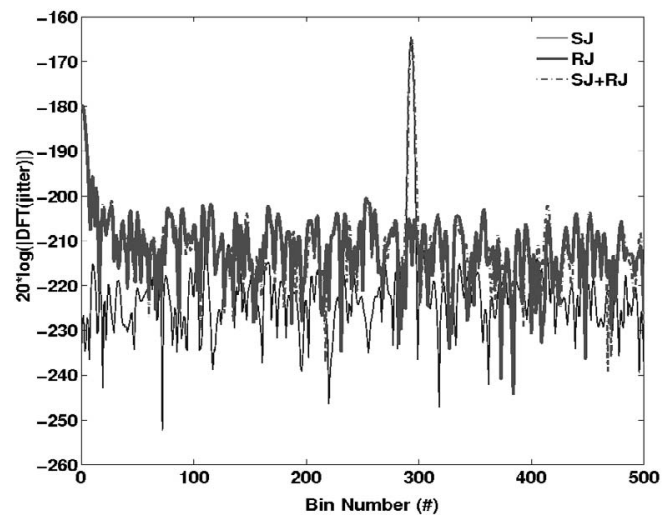

Fig. 11. DFT of the output jitter signal

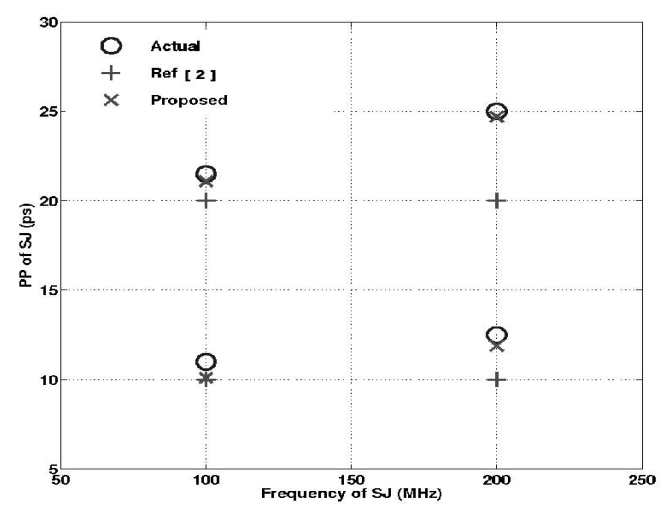

Fig. 12. Comparision of the PP values of the SJ

good agreement between theory and hspice simulation results was obtained. In future work we plan to apply this technique to estimate the BER. This requires separation of the DJ and RJ components in the absolute jitter.

\section{REFERENCES}

[1] A. Demir. Phase Noise and Timing Jitter in Oscillators with Colored-noise Sources. IEEE Trans. on Circuits and Systems I: Fundamental Theory and Applications, 49(12):1782-1791, Dec. 2002.

[2] F. Herzel and B. Razavi. A Study of Oscillator Jitter Due to Supply and Substrate Noise. IEEE Trans. on Circuits and Systems II: Analog and Digital Signal Processing, 46(1):56-62, Jan. 1999.

[3] D. Hong, C.-K. Ong, and K.-T. Cheng. BER Estimation for Serial Links Based on Jitter Spectrum and Clock Recovery Characteristics. In Proc. International Test Conference, pages 11381147. IEEE, Oct. 2004.

[4] M. Kossel and M. L. Schmatz. Jitter Measurements of HighSpeed Serial Links. Design \& Test of Computers, 21(6):536543, Nov. - Dec. 2004.

[5] M. Li, J. Wilstrup, R. Jessen, and D. Petrich. A New Method for Jitter Decomposition Through Its Distribution Tail Fitting. In Proc. International Test Conference, pages 788-794. IEEE, Sept. 1999.

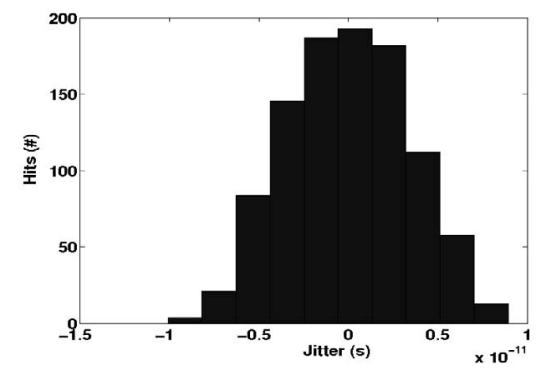

Fig. 13. Supply sinusoid noise $=1 \mathrm{mV}$

TABLE V

COMPARISION BETWEEN ACTUAL VALUES AND THE RESULTS FROM THE PROPOSED METHOD

\begin{tabular}{|l||l|l|l|l|l|l|}
\hline \multicolumn{1}{|c||}{} & \multicolumn{3}{c|}{ Actual Values } & \multicolumn{3}{c|}{ Proposed Method } \\
\hline $\begin{array}{l}V_{n} \\
(\mathrm{mV})\end{array}$ & $\begin{array}{l}f_{m} \\
(\mathrm{MHz})\end{array}$ & $\begin{array}{l}\text { PP } \\
(\mathrm{ps})\end{array}$ & $\begin{array}{l}\text { RMS } \\
(\mathrm{ps})\end{array}$ & $\begin{array}{l}f_{m} \\
(\mathrm{MHz})\end{array}$ & $\begin{array}{l}\text { PP } \\
(\mathrm{ps})\end{array}$ & $\begin{array}{l}\text { RMS } \\
(\mathrm{ps})\end{array}$ \\
\hline \hline 2.5 & 100 & 21.5 & 2.5 & 99.4 & 21.2 & 3.5 \\
1.25 & 100 & 11 & 2.5 & 97.2 & 10.1 & 3.4 \\
2.5 & 200 & 25 & 2.5 & 164.8 & 24.7 & 3.5 \\
1.25 & 200 & 12.5 & 2.5 & 161.8 & 11.9 & 3.4 \\
\hline
\end{tabular}

[6] C.-K. Ong, K.-T. C. Dongwoo Hong, and L.-C. Wang. A Scalable On-chip Jitter Extraction Technique. In Proc. VLSI Test Symposium, pages 267-272. IEEE, Apr. 2004.

[7] A. Papoulis. Probability, Random Variables and Stochastic Probability Processes. McGraw Hill, New York, 1984.

[8] K. Shanmugan and A. Breipohl. Random Signals: Detection, Estimation and Data Analysis. John Wiley \& Sons, Ontario, Canada, 1988.

[9] T. Yamaguchi, M. Ishida, M. Soma, K. Ichiyama, K. Christian, K. Ohsawa, and M. Sugai. A Real-time Jitter Measurement Board for High-performance Computer and Communication Systems. pages 77-84, Nov. 2004.

[10] C. Zhang, X. Wang, and L. Forbes. Simulation Technique for Noise and Timing Jitter in Electronic Oscillators. In Proc. Circuits, Devices and Systems, pages 184-189. IEEE, Apr. 2004. 